Polaroïd. L'impulsion d'amorçage de l'éclair photolytique est utilisée pour déclencher le balayage du tube cathodique. Pour éliminer le composant continu du signal du photomultiplicateur, on y oppose une tension étalonnée réglable (alimentation SAIP, $6 \mathrm{~V}$, taux de régulation $1 / 10000$ ). La figure 6 montre le schéma du circuit électrique associé au photo. multiplicateur.

\title{
BIBLIOGRAPHIE
}

[1] Kosower (E. M.) et LINDQvisT (L.), Tetrahedron Letters, 1965, 50, 4481.

[2] Lindovist (L.) et LUNDEEN (G. W.), J. Chem. Physics, 1966, 44, 1711.

[3] VIILAR (J.-G.) et LindQvist (L.), C. R. Acad. Sci., 1967, 264 B, 1807.

[4] Goldfarb (T.) et Lindquist (L.), J. Amer. Chem. Soc., 1967, 89, 4588.
[5] Kellmann (A.) et Lindqvist (L.), The Triplet State, ed. ZaHLAN (A. B.), Cambridge University Press, 1967, p. 439.

[6] LINDQvist (L.), Rev. Sci. Instrum., 1964, 35, 993.

[7] Claksson (S.) et Lindovist (L ), Arkiv Kemi, 1957, 11, 535 .

[8] Hatchard (C. G.) et Parker (C. A.), Proc. Roy. Soc., 1956, A 235, 518 .

\section{OBSERVATION OPTIQUE DES PERTES D'UN SUPRAGONDUGTEUR IMMERGÉ DANS L'HÉLIUM LIQUIDE}

\author{
Par M. COLOMBEAU, E. LE TIRAN, A. MAILFERT. \\ Laboratoire Central des Industries Électriques, Fontenay-aux-Roses. \\ (Reçu le 29 septembre 1967.)
}

\begin{abstract}
Résumé. - Les auteurs ont mis au point une méthode d'observation optique permettant de déceler de faibles échauffements d'un échantillon supraconducteur, placé dans l'hélium liquide, et traversé par un courant électrique alternatif.
\end{abstract}

Abstract. - An optical method is proposed by authors. It allows the observation of small temperature differences between a superconducting sample fed with an a.c. current, and the liquid helium bath surrounding it.

Dans un supraconducteur de deuxième espèce traversé par un courant électrique alternatif, il apparaît des pertes, qui sont mesurées habituellement par des méthodes calorimétriques [1] ou électriques [2]. Par exemple, pour du fil de Nb-25\% Zr, de diamètre $0,25 \mathrm{~mm}$, parcouru par un courant alternatif de fréquence $50 \mathrm{~Hz}$ et d'intensité efficace $60 \mathrm{~A}$, l'ordre de grandeur de ces pertes à $4,2{ }^{\circ} \mathrm{K}$ est $15 \mathrm{~mW} / \mathrm{m}$. Nous avons observé les variations d'indice optique d'un bain d'hélium liquide à $4,2{ }^{\circ} \mathrm{K}$ contenant un échantillon supraconducteur où existent des pertes. En effet, l'échange thermique entre le fil et le bain produit un échauffement et une variation d'indice des filets d'hélium situés à proximité de l'échantillon.

Appareillage. - L'appareil comprend un cryostat droit classique, dans lequel plongent deux tubes d'observation optique vidés, et munis chacun de trois disques de pyrex à faces parallèles (fig. 1). Le but des disques centraux, qui par leur position sont à la température de l'hélium liquide, est de servir d'écran pour le rayonnement émis par les disques supérieurs, de manière à éviter l'échauffement de l'hélium juste au-dessous des disques inférieurs.

La partie inférieure des tubes et l'échantillon sont placés dans un vase de pyrex métallisé ouvert à sa partie supérieure, et constituant ainsi une sorte de " vase à hélium tranquille », permettant de ne pas être gêné par les bulles de gaz prenant naissance au niveau des parois du cryostat.

Un système de miroirs plans permet d'envoyer sur l'échantillon un faisceau de lumière parallèle.

On a observé l'échantillon par deux méthodes différentes :

- Dans la première (observation directe), on utilise une lunette mise au point sur le fil. Celui-ci apparaît en sombre sur fond clair, et l'échauffement du fil se 


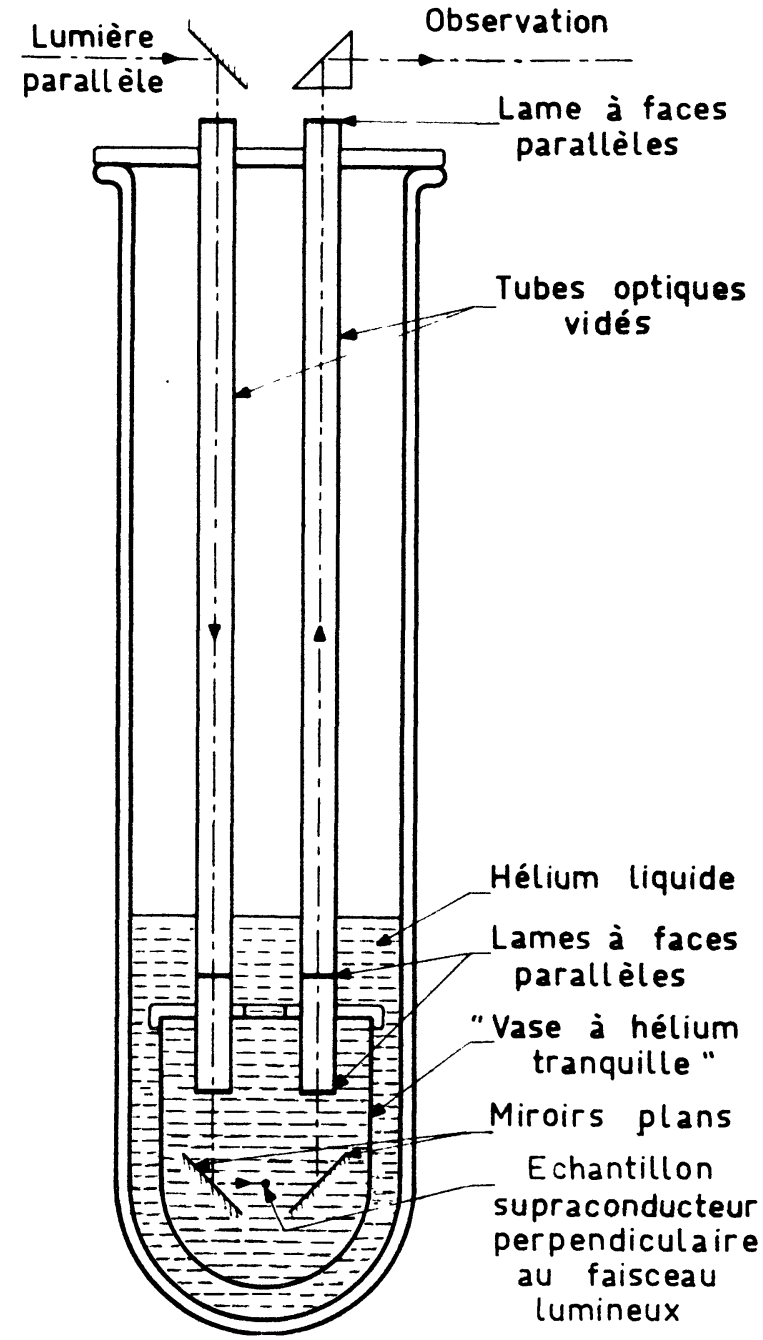

Frg. 1. - Appareillage (les arrivées de courant à l'échantillon supraconducteur ne sont pas représentées).

traduit (pour les faibles valeurs des pertes) par l'apparition de «fumées » sombres se détachant de l'échantillon.

- Dans la seconde (observation strioscopique), le faisceau parallèle est envoyé à la sortie du cryostat sur un miroir concave de grande distance focale $(1,50 \mathrm{~m})$. Au foyer du miroir, on place un cache de petites dimensions (de l'ordre de $0,5 \mathrm{~mm}$ de diamètre) qui a pour but d'arrêter tous les rayons lumineux non déviés lors du passage du faisceau parallèle dans le cryostat. Mais s'il y a eu des variations localisées de l'indice de réfraction au cours du trajet optique, le faisceau n'est plus parfaitement parallèle, et l'image obtenue au foyer du miroir n'est plus ponctuelle; les rayons déviés n'étant pas arrêtés par le cache, on peut observer, par exemple au moyen d'une lunette placée en arrière du cache sur l'axe optique, des variations d'indice du milieu. L'échantillon apparaît alors bril- lant sur fond sombre, et l'échauffement se traduit par des « fumées » claires sur fond sombre. Cette méthode utilisée avec succès dans des milieux tels que l'air [3] a en principe une sensibilité nettement plus élevée que l'observation directe en lumière parallèle.

Ces méthodes d'observation visuelles permettent un examen qualitatif (et, dans une faible mesure, quantitatif) des phénomènes de pertes ou d'échauffements dans l'hélium liquide.

Observations effectuées. - Nous avons observé le comportement d'un fil supraconducteur de Nb$25 \% \mathrm{Zr}$ de diamètre $0,25 \mathrm{~mm}$ parcouru par un courant de fréquence $50 \mathrm{~Hz}$. L'intensité critique efficace (cessation de la supraconductivité) est à cette fréquence $110 \mathrm{~A}$. La densité des «fumées 》 dues au phénomène de pertes rappelé précédemment est évidemment une fonction croissante de la puissance dissipée dans l'échantillon. Afin de tenter de mesurer quantitativement les pertes dans le supraconducteur, nous avons utilisé une méthode de comparaison. Nous avons utilisé comme élément de comparaison un fil de résistance connue, placé dans les mêmes conditions que le supraconducteur, et dans lequel on fait passer un courant connu : une même densité de «fumées » s'échappant du fil supraconducteur et du fil de comparaison doit correspondre à la même puissance dissipée. Il nous est apparu cependant difficile d'évaluer la densité des « fumées ». Seul leur seuil d'apparition, caractérisant la sensibilité de la méthode, semble assez bien défini. Nous avons ainsi trouvé pour le fil résistant un seuil d'apparition des «fumées » pour une puissance voisine de $20 \mathrm{~W} / \mathrm{m}^{-2}$ (la puissance est ramenée à l'unité de surface du fil en contact avec l'hélium liquide).

Le même seuil est obtenu lors du passage d'un courant de valeur efficace 60 A dans le fil supraconducteur, d'où l'on déduit que les pertes par unité de longueur dans le fil supraconducteur pour ce courant sont voisines de $15 \mathrm{~mW} / \mathrm{m}^{-1}$. Cette valeur est en accord avec des mesures effectuées par ailleurs avec une autre méthode sur le même matériau [4].

On peut se demander à quel régime d'échange thermique (convection libre, ébullition nucléée ou ébullition par film) correspond le seuil observé. Les courbes d'échange thermique [5], donnant la puissance dissipée par unité de surface en fonction de la différence de température entre un bain d'hélium liquide et une surface solide dans ce bain, indiquent que la valeur de $20 \mathrm{~W} / \mathrm{m}^{-2}$ correspond au début du régime d'ébullition nucléée et que la différence de température entre le matériau et le bain est alors de $0,06 \mathrm{oK}$.

Nous avons également observé qualitativement les conséquences d'un défaut localisé de l'échantillon, défaut obtenu en blessant mécaniquement le fil en un point. L'intensité critique efficace de l'échantillon après la blessure est 70 A. Nous avons observé les 
premières «fumées » traduisant un échauffement localisé de la partie blessée pour une intensité efficace de $35 \mathrm{~A}$.

Conclusion. - Les méthodes d'observation optique que nous venons de décrire permettent de mettre en évidence qualitativement des échauffements de quelques centièmes de degré d'échantillons placés dans l'hélium liquide. Une mesure quantitative ne semble possible qu'en utilisant une méthode de comparaison, et dans ce cas l'observation visuelle ne permet pas une mesure de grande précision; l'utilisation de cellules photoélectriques permettrait peut-être des mesures comparatives plus précises.

Nous avons obtenu la même sensibilité par les deux méthodes optiques décrites dans cet article, mais nous pensons que l'amélioration de la qualité optique de notre montage permettrait d'accroître la sensibilité de la méthode strioscopique.

Dans le cas des pertes dans les supraconducteurs, la méthode optique permet d'observer des échauffements localisés des échantillons, ce qui n'est pas possible par les méthodes électriques ou calorimétriques habituelles. On peut espérer ainsi déceler par exemple des fractions défectueuses dans de grandes longueurs de fil supraconducteur.

Les auteurs remercient M. le Professeur Fournet pour des discussions fructueuses, et M. Carrie qui a réalisé les tubes d'observation optique.

Ce travail a été effectué avec le soutien de la D.G.R.S.T. (Gomité Électrotechnique Nouvelle).

\section{BIBLIOGRAPHIE}

[1] JONES (C. H.) et SCHENK (H. L.), Advances in cryogenic engineering, vol. 8, p. 579.

RHOdES (R. G.), ROGERS (E. C.) et SEEHOLD (R. J. A.), Cryogenics, 1964, 4, 206

[2] Pech (T.), Duflot (J. P.) et Fournet (G.), Phys. Letters, 1965, 16, 201.

Damman (C.), Santamaria (E.), Maldy (J.) et Donadieu (L.), Phys. Letters, 1967, 24 A, 574.
[3] Fayolle (P.) et NASLIN (P.), Photographie instantanée et cinématographie ultrarapide, éd. Revue d'Optique, Paris, 1950.

[4] Claude (M. L.) et MailfFert (A.), Phys. Letters, 1967, 24 A, 150 .

[5] DOREY (A. P.), Cryogenics, juin 1965, 5, 146. 